\title{
THE FLOW OF A NON-NEWTONIAN FLUID INDUCED DUE TO THE OSCILLATIONS OF A POROUS PLATE
}

\author{
S. ASGHAR, M. R. MOHYUDDIN, T. HAYAT, AND A. M. SIDDIQUI \\ Received 1 September 2003 and in revised form 9 February 2004
}

An analytic solution of the flow of a third-grade fluid on a porous plate is constructed. The porous plate is executing oscillations in its own plane with superimposed injection or suction. An increasing or decreasing velocity amplitude of the oscillating porous plate is also examined. It is also shown that in case of third-grade fluid, a combination of suction/injection and decreasing/increasing velocity amplitude is possible as well. Several limiting situations with their implications are given and discussed.

\section{Introduction}

Mechanics of nonlinear fluids present a special challenge to engineers, physicists, and mathematicians. The nonlinearity can manifest itself in a variety of ways. One of the simplest ways in which the viscoelastic fluids have been classified is the methodology given by Rivlin and Ericksen [31] and Truesdell and Noll [35], who present constitutive relations for the stress tensor as a function of the symmetric part of the velocity gradient and its higher (total) derivatives.

In recent years, there have been several studies $[1,5,13,14,15,18,25,33]$ on flows of non-Newtonian fluids, not only because of their technological significance, but also in view of the interesting mathematical features presented by the equations governing the flow. On the other hand, it is well known that the rheological properties of many fluids are not well modelled by the Navier-Stokes equations. For example, in most of these models, a significant reduction of the drag past solid walls has been observed. Moreover, elastic properties of real fluids can be detected and measured. A discussion of the various differential, rate-type, and integral models can be found in the books of Schowalter [32] and Huilgol [19], and in the survey article by Rajagopal [24]. Amongst these models, fluids of differential type have attracted much attention, as well as much controversy. We refer the reader to Dunn and Rajagopal [7] for a complete and thorough discussion of all the relevant issues. The major attractiveness of these models is the fact that the constitutive relations, whether we take the second- or the third-grade fluids since they 
have been studied the most, are derived based on first principles, and unlike many other "phenomenological" models [30], there are no curve fittings or parameters to adjust. Though, in both of these grade models, there are material properties that need to be measured. At the same time, the sign of these material parameters and the stability or instability of the motions have caused a certain degree of misunderstanding. These issues for the second- and third-grade fluids have been discussed in detail by Dunn and Fosdick [6], and Fosdick and Rajagopal [11], respectively.

In general, for fluids of the differential type of grade $n$, the equations of motion are of order $(n+1)$. Thus, if $n>1$, then the adherence boundary condition is insufficient for determinacy. The standard method used to overcome this difficulty is to resort to perturbation that lowers the order of the equation $[2,4,8,12,27,29,34]$, which is not mathematically rigorous. In fact, the authors in $[2,4,8,12,27,29,34]$ are aware of this, but in the absence of any rational method for generating additional boundary conditions, they have no other way out of the impasse. It is possible that in flows in unbounded domains, we can obtain additional conditions based on the asymptotic structure of the flow at infinity. Mansutti et al. [22] showed that results by perturbation method and of augmenting the boundary conditions agree remarkably well. Rajagopal and Gupta have also discussed this issue in [26] and studied the steady flow of a second-grade fluid past a porous plate. In another paper, Rajagopal [23] studied some unidirectional flows of a second-grade fluid. In [10], Foote et al. studied the problem for the flow of an elasticoviscous fluid on an oscillating porous plate. More recently, Turbatu et al. [36] discussed the viscous flow of an oscillating porous flat plate with the combination of superimposed injection or suction and increasing or decreasing velocity amplitude. Hayat et al. [16] extended this analysis for the viscous flow caused by the noncoaxial rotations of a porous oscillating disk and a fluid at infinity.

Although the second-grade fluid model is able to predict the normal stress differences which are characteristic of non-Newtonian fluids, it does not take the shear thinning and thickening phenomena that many show.

The third-grade fluid model represents a further, although inconclusive, attempt toward a comprehensive description of the properties of viscoelastic fluids. With this in view, the model in the present paper is a third-grade fluid one. Related studies are in $[3,9,28]$.

The task of this work is to study the unsteady flow of a third-grade fluid on an oscillating porous plate with the combination of superimposed suction or blowing and decreasing or increasing velocity amplitude. In case of third-grade fluid also, a combination of suction or injection and decreasing or increasing velocity amplitude of the oscillating plate is possible. It is found that with the increase in material parameters of the third-grade fluid, the velocity boundary layer thickness decreases in the case of suction and increases in the case of blowing and the amplitude of oscillation decreases for acceleration and increases for deceleration. A comparison of the third-grade fluid with the viscous case shows that in the case of third-grade fluid, the rate of change of boundary layer thickness decrease/increase for suction/blowing and the rate of change of amplitude of the oscillation increase/decrease for acceleration/deceleration are greater than that for the viscoelastic [17] and viscous [36] cases. 


\section{Basic equations}

The stress in a third-grade fluid is given by [35]

$$
\mathbf{T}=-p \mathbf{I}+\mu \mathbf{A}_{1}+\alpha_{1} \mathbf{A}_{2}+\alpha_{2} \mathbf{A}_{1}^{2}+\beta_{1} \mathbf{A}_{3}+\beta_{2}\left(\mathbf{A}_{2} \mathbf{A}_{1}+\mathbf{A}_{1} \mathbf{A}_{2}\right)+\beta_{3}\left(\operatorname{tr} \mathbf{A}_{2}\right) \mathbf{A}_{1},
$$

where $\mu$ is the coefficient of viscosity, and $\alpha_{1}, \alpha_{2}, \beta_{1}, \beta_{2}$, and $\beta_{3}$ are the material moduli. In the above representation, $-p \mathbf{I}$ is the spherical stress due to the constraint of incompressibility, and the kinematic tensors $\mathbf{A}_{1}, \mathbf{A}_{2}$, and $\mathbf{A}_{3}$ are defined by

$$
\begin{gathered}
\mathbf{A}_{1}=(\operatorname{grad} \mathbf{V})+(\operatorname{grad} \mathbf{V})^{\top}, \\
\mathbf{A}_{n}=\frac{d \mathbf{A}_{n-1}}{d t}+\mathbf{A}_{n-1}(\operatorname{grad} \mathbf{V})+(\operatorname{grad} \mathbf{V})^{\top} \mathbf{A}_{n-1}, \quad n=2,3,
\end{gathered}
$$

where $\mathbf{V}$ denotes the velocity field, grad is the gradient operator, $T$ the transpose, and $d / d t$ is the material time derivative which is defined by

$$
\frac{d}{d t}(\cdot)=\frac{\partial}{\partial t}(\cdot)+[\operatorname{grad}(\cdot)] \mathbf{V}
$$

where $\partial / \partial t$ is the partial derivative with respect to time. A detailed thermodynamic analysis of the model, represented by (2.1), is given by Fosdick and Rajagopal [11]. They showed that if all the motions of the fluid are to be compatible with thermodynamics in the sense that these motions meet the Clausius-Duhem inequality and if it is assumed that the specific Helmholtz free energy is a minimum when the fluid is locally at rest, then

$$
\mu \geq 0, \quad \alpha_{1} \geq 0, \quad \beta_{1}=\beta_{2}=0, \quad \beta_{3} \geq 0, \quad\left|\alpha_{1}+\alpha_{2}\right| \leq \sqrt{24 \mu \beta_{3}} .
$$

It is pointed out that the second-grade fluid is incapable of shear thinning or shear thickening. The third-grade fluid model represented by (2.1), under the assumption given in (2.4), is a shear thickening fluid. Rigorous mathematical results have been established for fluids that can shear thin or shear thicken in the book by Málek et al. [20]. Also, the issues concerning mathematical results, especially concerning the stability of flows of fluids that can shear thicken or shear thin, are discussed by Málek et al. [21]. The constitutive relation for a thermodynamically compatible third-grade fluid becomes

$$
\mathbf{T}=-p \mathbf{I}+\mu \mathbf{A}_{1}+\alpha_{1} \mathbf{A}_{2}+\alpha_{2} \mathbf{A}_{1}^{2}+\beta_{3}\left(\operatorname{tr} \mathbf{A}_{2}\right) \mathbf{A}_{1} .
$$

If the normal stress parameters $\alpha_{1}$ and $\alpha_{2}$ are zero, then

$$
\mathbf{T}=-p \mathbf{I}+\left[\mu+\beta_{3}\left(\operatorname{tr} \mathbf{A}_{2}\right)\right] \mathbf{A}_{1},
$$

where the quantity in the brackets can be thought of as an effective shear-dependent viscosity. If we go to (2.1) and assume $\beta_{1}=\beta_{2}=\beta_{3}=0$, we obtain the model for the second-grade fluid:

$$
\mathbf{T}=-p \mathbf{I}+\mu \mathbf{A}_{1}+\alpha_{1} \mathbf{A}_{2}+\alpha_{2} \mathbf{A}_{1}^{2}
$$

This model also has a constant shear viscosity. 


\section{Statement of the problem}

We are interested in the third-grade fluid over an infinite plate. The plate considered is porous. Choose the $x$-axis along the plate and the $y$-axis perpendicular to it. For $t<0$, fluid is at rest and for $t>0$, the plate is moving periodically. The basic governing equations are the conservation of mass and the conservation of linear momentum. These are

$$
\begin{aligned}
& \frac{\partial \rho}{\partial t}+\operatorname{div}(\rho \mathbf{V})=0, \\
& \rho \frac{d \mathbf{V}}{d t}=\operatorname{div} \mathbf{T}+\rho \mathbf{b},
\end{aligned}
$$

where $\rho$ is the density and $\mathbf{b}$ is the body force. Since we are assuming that the fluid can undergo only isochoric motion, (3.1) reduces to

$$
\operatorname{div} \mathbf{V}=0 .
$$

We seek a solution for the velocity field of the form

$$
u=u(y, t), \quad v=V_{0},
$$

where $u$ and $v$ are velocity components in the $x$-and $y$-coordinates direction, respectively. Also $V_{0}<0$ is the suction velocity and $V_{0}>0$ is the blowing velocity.

The boundary conditions on the flow are

$$
\begin{aligned}
& u(0, t)=U(t)= U_{0} \cdot e^{\left(\beta_{0}-i \omega\right) t}, \quad \omega>0, t>0, \beta_{0}=\text { const } \neq 0, \\
& u(y, t) \longrightarrow 0 \quad \text { as } y \longrightarrow \infty,
\end{aligned}
$$

where $U_{0}$ is the reference velocity or free-stream velocity.

Using (3.4), (3.3) is identically satisfied and (3.2) in the absence of body forces yields

$$
\frac{\partial u}{\partial t}+V_{0} \frac{\partial u}{\partial y}=\nu \frac{\partial^{2} u}{\partial y^{2}}+\beta\left[V_{0} \frac{\partial^{3} u}{\partial y^{3}}+\frac{\partial^{3} u}{\partial y^{2} \partial t}\right]+\gamma\left[\frac{\partial^{2} u}{\partial y^{2}}\left(\frac{\partial u}{\partial y}\right)^{2}\right],
$$

where

$$
\nu=\frac{\mu}{\rho}, \quad \beta=\frac{\alpha_{1}}{\rho}, \quad \gamma=\frac{6 \beta_{3}}{\rho} .
$$

The shear stress in the direction of $x$-axis normal to the $y$-axis is given by

$$
\tau_{x y}=\mu \frac{\partial u}{\partial y}+\alpha_{1}\left\{V_{0} \frac{\partial^{2} u}{\partial y^{2}}+\frac{\partial^{2} u}{\partial y \partial t}\right\}+2 \beta_{3}\left(\frac{\partial u}{\partial y}\right)^{3} .
$$

The above equations can be rewritten in the following dimensionless form:

$$
\begin{gathered}
\frac{\partial f}{\partial \tau}+\sqrt{2} d \frac{\partial f}{\partial \eta}=\frac{1}{2} \frac{\partial^{2} f}{\partial \eta^{2}}+\phi_{1}\left[\frac{\partial^{3} f}{\partial \eta^{2} \partial \tau}+\sqrt{2} d \frac{\partial^{3} f}{\partial \eta^{3}}\right]+\phi_{2} \frac{\partial^{2} f}{\partial \eta^{2}}\left(\frac{\partial f}{\partial \eta}\right)^{2}, \\
\bar{\tau}_{x y}=\frac{1}{\sqrt{2 \nu \omega} \rho U_{0}} \tau_{x y}=\frac{1}{2} \frac{\partial f}{\partial \eta}+\phi_{1}\left[\frac{\partial^{2} f}{\partial \eta \partial \tau}+\sqrt{2} d \frac{\partial^{2} f}{\partial \eta^{2}}\right]+\frac{1}{2} \phi_{2}\left(\frac{\partial f}{\partial \eta}\right)^{3},
\end{gathered}
$$


where

$$
\begin{aligned}
& \eta=\sqrt{\frac{\omega}{2 v}} y, \quad d=\frac{V_{0}}{2 \sqrt{v \omega}}, \quad \tau=\omega t, \\
& f=\frac{u}{U_{0}}, \quad \phi_{1}=\frac{\omega}{2 v} \beta, \quad \phi_{2}=\frac{\omega U_{0}^{2}}{4 v^{2}} \gamma .
\end{aligned}
$$

The appropriate boundary conditions are

$$
\begin{gathered}
f(0, \tau)=e^{(c-i) \tau}, \quad c=\frac{\beta_{0}}{\omega}, \\
f \longrightarrow 0 \text { as } \eta \longrightarrow \infty
\end{gathered}
$$

\section{Solution of the problem}

We suppose the dimensionless velocity $f$ can be expanded in power series in $\phi_{2}$ [28]:

$$
f\left(\eta, \tau ; \phi_{2}\right)=f_{0}(\eta, \tau)+\phi_{2} f_{1}(\eta, \tau)+\cdots
$$

On substituting the expansion (4.1) for $f$ and equating like powers of $\phi_{2}$, we obtain the following equations of zeroth and first powers, respectively:

$$
\begin{gathered}
\frac{\partial f_{0}}{\partial \tau}+\sqrt{2} d \frac{\partial f_{0}}{\partial \eta}=\frac{1}{2} \frac{\partial^{2} f_{0}}{\partial \eta^{2}}+\phi_{1}\left[\frac{\partial^{3} f_{0}}{\partial \eta^{2} \partial \tau}+\sqrt{2} d \frac{\partial^{3} f_{0}}{\partial \eta^{3}}\right], \\
f_{0}(0, \tau)=e^{(c-i) \tau}, \\
f_{0}(\eta, \tau) \longrightarrow 0 \quad \text { as } \eta \longrightarrow \infty, \\
\frac{\partial f_{1}}{\partial \tau}+\sqrt{2} d \frac{\partial f_{1}}{\partial \eta}=\frac{1}{2} \frac{\partial^{2} f_{1}}{\partial \eta^{2}}+\phi_{1}\left[\frac{\partial^{3} f_{1}}{\partial \eta^{2} \partial \tau}+\sqrt{2} d \frac{\partial^{3} f_{1}}{\partial \eta^{3}}\right]+\frac{\partial^{2} f_{0}}{\partial \eta^{2}}\left(\frac{\partial f_{0}}{\partial \eta}\right)^{2}, \\
f_{1}(0, \tau)=0, \\
f_{1}(\eta, \tau) \longrightarrow 0 \quad \text { as } \eta \longrightarrow \infty .
\end{gathered}
$$

Introducing the similarity transformations

$$
f_{0}(\eta, \tau)=g_{0}(\eta) e^{(c-i) \tau}, \quad f_{1}(\eta, \tau)=g_{1}(\eta) e^{3(c-i) \tau}
$$

in (4.2), and employing the procedure used in [2], we obtain the following real parts:

$$
\begin{aligned}
f_{0}(\eta, \tau)= & \exp \left[c \tau+\left(C_{0 R}+C_{1 R} \phi_{1}+C_{2 R} \phi_{1}^{2}\right) \eta\right] \\
& \times \cos \left[\tau-\left(C_{0 I}+C_{1 I} \phi_{1}+C_{2 I} \phi_{1}^{2}\right) \eta\right], \\
f_{1}(\eta, \tau)= & e^{\left(3 c \tau+k_{3} \eta\right)}\left[q_{1} \cos \left(3 \tau-k_{4} \eta\right)+q_{2} \sin \left(3 \tau-k_{4} \eta\right)\right] \\
& -e^{3\left(c \tau+k_{1} \eta\right)}\left[q_{1} \cos 3\left(\tau-k_{2} \eta\right)+q_{2} \sin 3\left(\tau-k_{2} \eta\right)\right],
\end{aligned}
$$

where the constants appearing in (4.4), and in the forthcoming special cases of (4.4), can be found in a straight manner. The velocity field can be obtained by combining the results 
(4.4) in (4.1). The dimensionless stress at the plate $(\eta=\sqrt{\omega / 2 v} y=0)$ is respectively given by

$$
\begin{aligned}
\bar{\tau}_{0 w}= & \frac{1}{\sqrt{2 \nu \omega} \rho U_{0}} \tau_{0 w}=\frac{1}{2} A \phi_{1} e^{(c-i) \tau}[\sqrt{2} d A+(c-i)] \\
\bar{\tau}_{1 w}= & \frac{1}{\sqrt{2 \nu \omega} \rho U_{0}} \tau_{1 w} \\
= & \frac{A^{4} e^{3(c-i) \tau}}{A^{*}}\left[B\left\{\frac{1}{2}+(\sqrt{2} d B+3(c-i)) \phi_{1}\right\}-A\left\{\frac{3}{2}+9(A+c-i) \phi_{1}\right\}\right] \\
& +\frac{1}{3} A^{3} e^{3(c-i) \tau} .
\end{aligned}
$$

\section{Special cases}

To understand the different physical aspects of solution (4.1), we discuss some special cases.

5.1. Oscillating plate (viscous; $c=d=\phi_{1}=\phi_{2}=0$ ). Stokes' second problem can be obtained by taking $c=d=\phi_{1}=\phi_{2}=0$, that is,

$$
f_{N S}(\eta, \tau)=\exp (-\eta) \cos (\tau-\eta)
$$

5.2. Oscillating porous plate (viscous; $c \neq 0, d \neq 0$, and $\phi_{1}=\phi_{2}=0$ ). The results of Turbatu et al. [36] can be readily recovered by taking $c \neq 0, d \neq 0, \phi_{1}=\phi_{2}=0$, that is,

$$
f_{N P}(\eta, \tau)=\exp \left[c \tau+C_{0 R} \eta\right] \cos \left[\tau-C_{0 I} \eta\right]
$$

5.3. Oscillating porous plate (viscoelastic; $c \neq 0, d \neq 0, \phi_{1} \neq 0$, and $\phi_{2}=0$ ). Viscoelastic second-grade fluid [17] can be obtained by taking $c \neq 0, d \neq 0, \phi_{1} \neq 0$, and $\phi_{2}=0$, that is,

$$
\begin{aligned}
f_{V}(\eta, \tau)= & \exp \left[c \tau+\left(C_{0 R}+C_{1 R} \phi_{1}+C_{2 R} \phi_{1}^{2}\right) \eta\right] \\
& \times \cos \left[\tau-\left(C_{0 I}+C_{1 I} \phi_{1}+C_{2 I} \phi_{1}^{2}\right) \eta\right] .
\end{aligned}
$$

5.4. Oscillating porous plate (third grade; $c=0, d \neq 0, \phi_{1} \neq 0$, and $\phi_{2} \neq 0$ ). For $c=0$, $d \neq 0, \phi_{1} \neq 0$, and $\phi_{2} \neq 0$, solution (4.1) gives

$$
\begin{aligned}
f(\eta, \tau)= & \exp \left[\left(\hat{C}_{0 R}+\hat{C}_{1 R} \phi_{1}+\hat{C}_{2 R} \phi_{1}^{2}\right) \eta\right] \\
& \times \cos \left[\tau-\left(\hat{C}_{0 I}+\widehat{C}_{1 I} \phi_{1}+\hat{C}_{2 I} \phi_{1}^{2}\right) \eta\right] \\
+ & \phi_{2}\left\{e^{\left(3 c \tau+\hat{k}_{3} \eta\right)}\left[\hat{q}_{1} \cos \left(3 \tau-\hat{k}_{4} \eta\right)+\hat{q}_{2} \sin \left(3 \tau-\hat{k}_{4} \eta\right)\right]\right. \\
& \left.\quad-e^{3\left(c \tau+\hat{k}_{1} \eta\right)}\left[\hat{q}_{1} \cos 3\left(\tau-\hat{k}_{2} \eta\right)+\hat{q}_{2} \sin 3\left(\tau-\hat{k}_{2} \eta\right)\right]\right\} .
\end{aligned}
$$

The solution for the velocity component $f$ is plotted in Figures 5.1 and 5.2 for different values of $\phi_{1}$ and $\phi_{2}$ and for a fixed time $\tau=2 \pi$ as a function of the suction/blowing velocity $V_{0}$, given by $d=V_{0} / 2 \sqrt{v \omega}$. The values $d=0, \phi_{1}=0$, and $\phi_{2}=0$ refer to the 


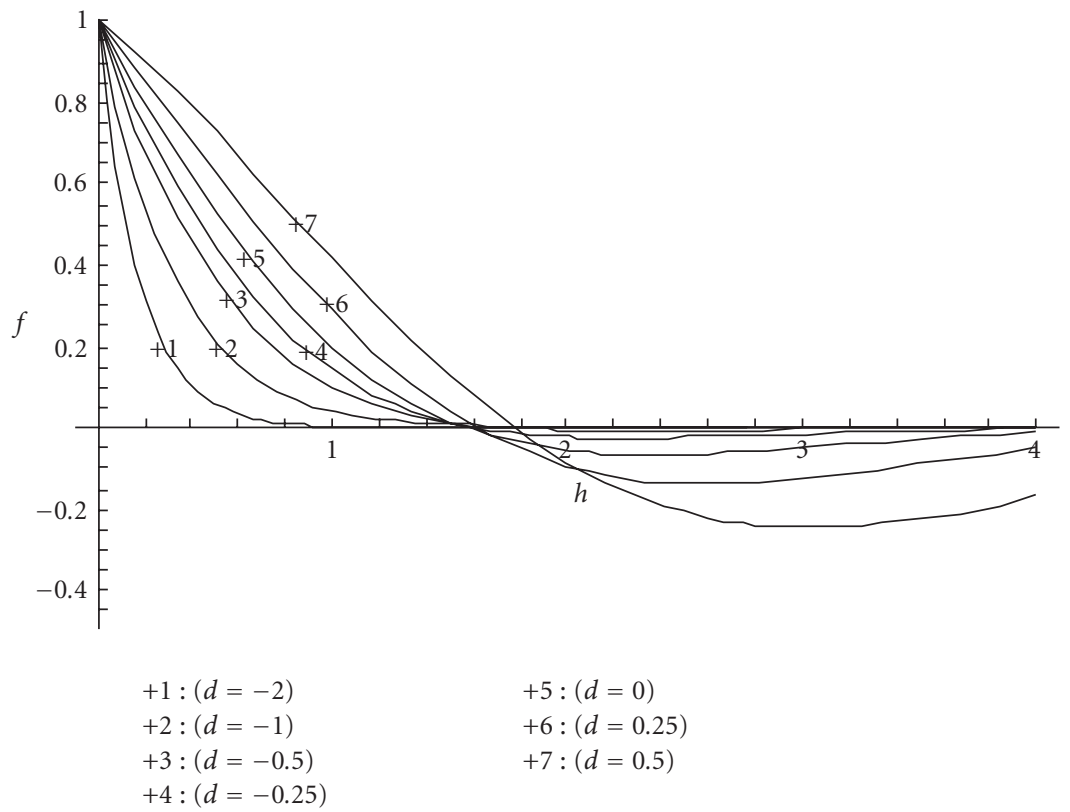

Figure 5.1. Influence of suction/blowing on the velocity distribution at $\tau=2 \pi ; c=0, \phi_{1}=0, \phi_{2}=0$.

classical Stokes problem. It is noted that the boundary layer thickness is controlled by the suction velocity $\left(V_{0}<0\right)$, that is, it decreases with an increase in suction velocity.

In case of blowing $\left(V_{0}>0\right)$, the boundary layer thickness becomes large as is expected physically.

Figure 5.2 gives the effect of material parameter of third-order fluid. It is observed that with the increase in third-grade parameter $\phi_{2}$, the boundary layer thickness rapidly decreases in the case of suction $\left(V_{0}<0\right)$ and rapidly increases in the case of blowing $\left(V_{0}>0\right)$ when compared with the viscoelastic case [17] and viscous case [36].

5.5. Oscillating plate with acceleration/deceleration (third grade; $c \neq 0, d=0, \phi_{1} \neq 0$, and $\left.\phi_{2} \neq 0\right)$. In this section, the superposition of two time-dependent functions is taken into account, one of which is due to the oscillation of the plate with imposed frequency $\omega$ and the second is an exponential increase or decrease of the velocity amplitude of the plate with the parameter $\beta_{0}$.

For $d=0, c \neq 0, \phi_{1} \neq 0$, and $\phi_{2} \neq 0$, solution (4.1) takes the form

$$
\begin{aligned}
f(\eta, \tau)= & \exp \left[c \tau+\left(\widetilde{C}_{0 R}+\widetilde{C}_{1 R} \phi_{1}+\widetilde{C}_{2 R} \phi_{1}^{2}\right) \eta\right] \\
& \times \cos \left[\tau-\left(\widetilde{C}_{0 I}+\widetilde{C}_{1 I} \phi_{1}+\widetilde{C}_{2 I} \phi_{1}^{2}\right) \eta\right] \\
& +\phi_{2}\left\{e^{\left(3 c \tau+\tilde{k}_{3} \eta\right)}\left[\tilde{q}_{1} \cos \left(3 \tau-\tilde{k}_{4} \eta\right)+\tilde{q}_{2} \sin \left(3 \tau-\tilde{k}_{4} \eta\right)\right]\right. \\
& \left.\quad-e^{3\left(c \tau+\tilde{k}_{1} \eta\right)}\left[\tilde{q}_{1} \cos 3\left(\tau-\tilde{k}_{2} \eta\right)+\tilde{q}_{2} \sin 3\left(\tau-\tilde{k}_{2} \eta\right)\right]\right\},
\end{aligned}
$$




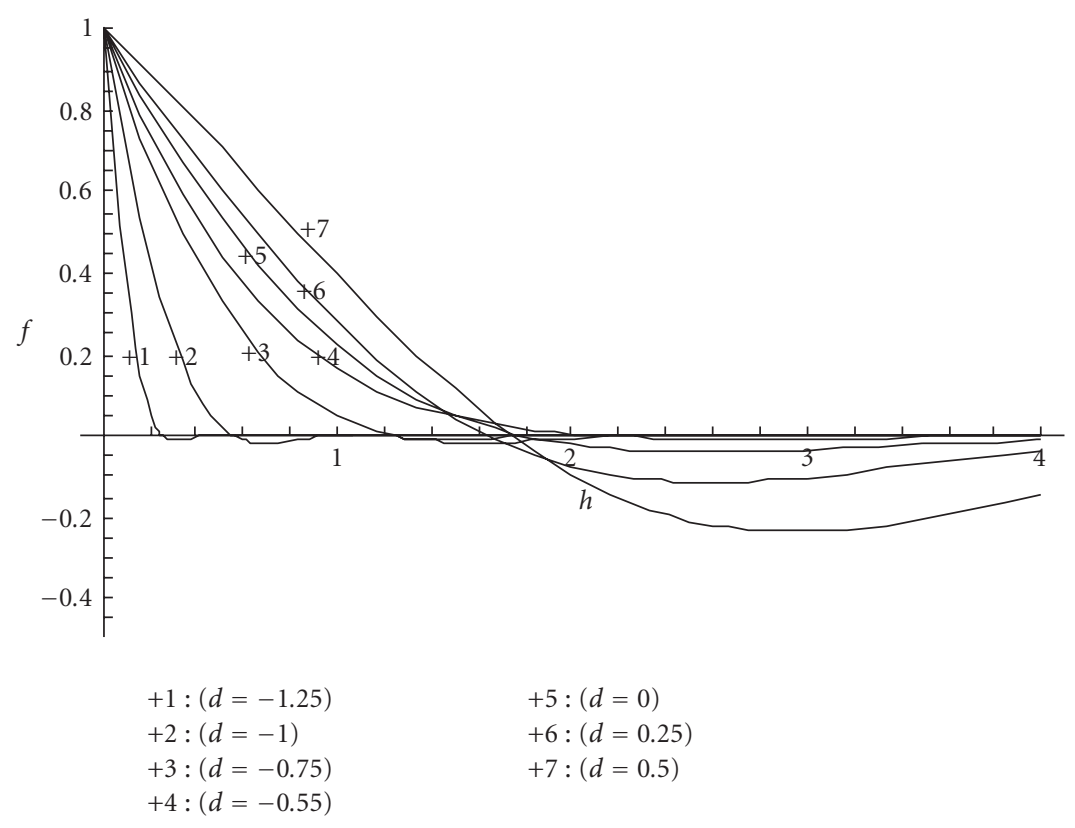

Figure 5.2. Influence of suction/blowing on the velocity distribution at $\tau=2 \pi ; c=0, \phi_{1}=0.1, \phi_{2}=$ 0.1 .

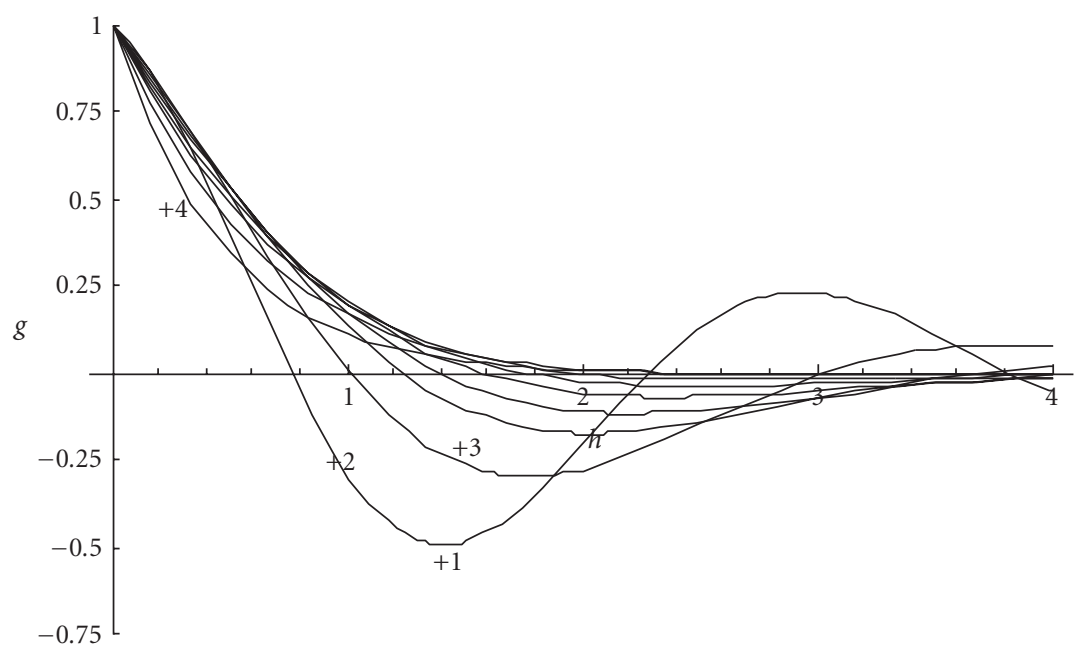

$$
\begin{aligned}
& +1:(c=-2) \\
& +2:(c=-1) \\
& +3:(c=-0.5) \\
& +4:(c=2)
\end{aligned}
$$

Figure 5.3. Influence of increasing or decreasing the amplitude of the plate on the normalized velocity distribution at $\tau=2 \pi ; d=0, \phi_{1}=0, \phi_{2}=0$. 


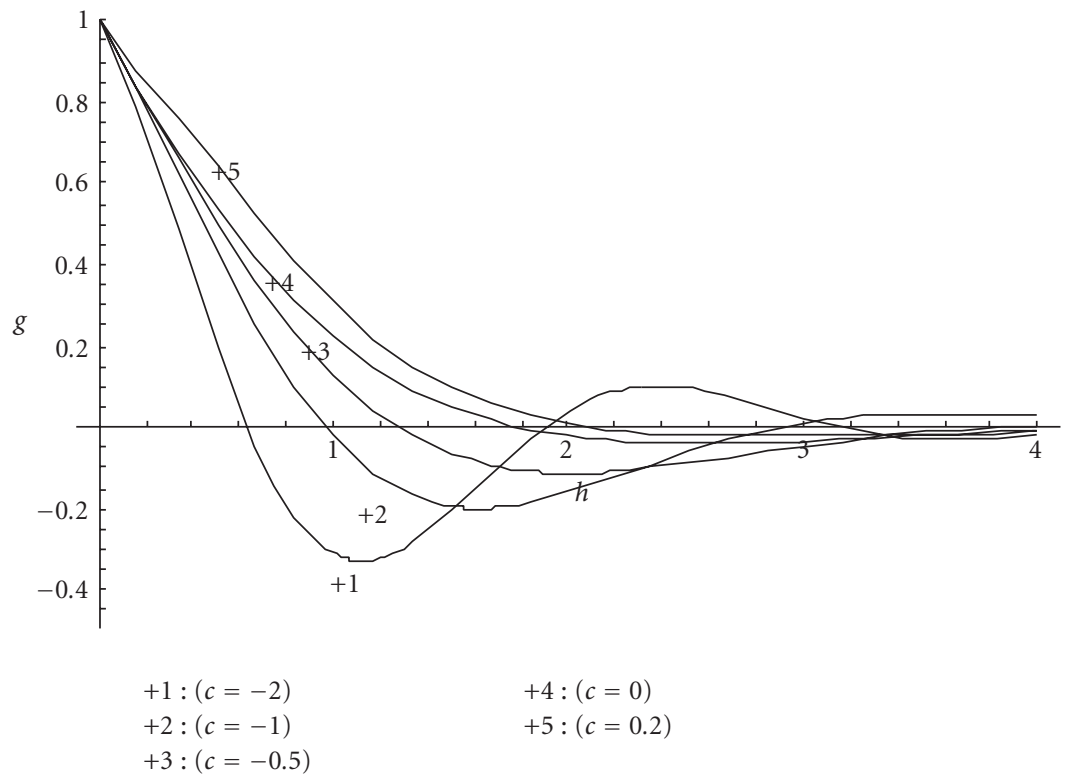

Figure 5.4. Influence of increasing or decreasing the amplitude of the plate on the normalized velocity distribution at $\tau=2 \pi ; d=0, \phi_{1}=0.1, \phi_{2}=0.1$.

or

$$
\begin{aligned}
& g(\eta, \tau)=\frac{f(\eta, \tau)}{\exp (c \tau)}= \exp \left[\left(\widetilde{C}_{0 R}+\widetilde{C}_{1 R} \phi_{1}+\widetilde{C}_{2 R} \phi_{1}^{2}\right) \eta\right] \\
& \times \cos \left[\tau-\left(\widetilde{C}_{0 I}+\widetilde{C}_{1 I} \phi_{1}+\widetilde{C}_{2 I} \phi_{1}^{2}\right) \eta\right] \\
&+\phi_{2}\left\{e^{\left(2 c \tau+\tilde{k}_{3} \eta\right)}\left[\tilde{q}_{1} \cos \left(3 \tau-\tilde{k}_{4} \eta\right)+\tilde{q}_{2} \sin \left(3 \tau-\tilde{k}_{4} \eta\right)\right]\right. \\
&\left.\quad-e^{\left(2 c \tau+3 \tilde{k}_{1} \eta\right)}\left[\tilde{q}_{1} \cos 3\left(\tau-\tilde{k}_{2} \eta\right)+\tilde{q}_{2} \sin 3\left(\tau-\tilde{k}_{2} \eta\right)\right]\right\} .
\end{aligned}
$$

The parameter $c=\beta_{0} / \omega$ gives the variation of the amplitude of the plate velocity and $c=0, \phi_{1}=0$, and $\phi_{2}=0$ imply the classical viscous case. Solution (5.6) is plotted in Figures 5.3 and 5.4 for $\tau=2 \pi, \phi_{1}=0, \phi_{2}=0$, and for $\tau=2 \pi, \phi_{1}=0.1, \phi_{2}=0.1$, respectively. Figures 5.3 and 5.4 show the variation of $\beta_{0}, \phi_{1}$, and $\phi_{2}$. It is noted that with the increase in third-grade parameter $\phi_{2}$, the amplitude of the oscillations rapidly increases/decreases according to $\beta_{0}>0 / \beta_{0}<0$.

\section{Conclusion}

We have presented here results for the flow field of a fluid, which is called the third-order fluid or the fluid of grade three, on an oscillating plate with superimposed blowing or suction. The analysis presented is further concerned with an increasing or decreasing velocity amplitude of the oscillating plate. The results in $[17,36]$ can be recovered as a special case of the problem considered by taking the parameters $\phi_{1}$ and $\phi_{2}$ to be zero and $\phi_{2}$ to be zero, respectively. This provides a useful mathematical check. 


\section{References}

[1] M. Akçay and M. A. Yükselen, Drag reduction of a non-Newtonian fluid by fluid injection on a moving wall, Arch. Appl. Mech. 69 (1999), no. 3, 215-225.

[2] J. Astin, R. S. Jones, and P. Lockyer, Boundary layers in non-Newtonian fluids, J. Mecanique 12 (1973), 527-539.

[3] R. Bandelli, I. Lapczyk, and H. Li, Longitudinal and torsional oscillations of a rod in a third-grade fluid, Internat. J. Non-Linear Mech. 29 (1994), no. 3, 397-408.

[4] D. W. Beard and K. Walters, Elastico-viscous boundary-layer flows. I. Two-dimensional flow near a stagnation point, Proc. Cambridge Philos. Soc. 60 (1964), 667-674.

[5] A. M. Benharbit and A. M. Siddiqui, Certain solutions of the equations of the planar motion of a second grade fluid for steady and unsteady cases, Acta Mech. 94 (1992), no. 1-2, 85-96.

[6] J. E. Dunn and R. L. Fosdick, Thermodynamics, stability, and boundedness of fluids of complexity 2 and fluids of second grade, Arch. Rational Mech. Anal. 56 (1974), 191-252.

[7] J. E. Dunn and K. R. Rajagopal, Fluids of differential type: critical review and thermodynamic analysis, Internat. J. Engrg. Sci. 33 (1995), no. 5, 689-729.

[8] M. E. Erdogan, On the flow of a non-Newtonian fluid past a porous flat plate, Z. Angew. Math. Mech. 55 (1975), 99-103.

[9] - Plane surface suddenly set in motion in a non-Newtonian fluid, Acta Mech. 108 (1995), no. $1-4,179-187$.

[10] J. R. Foote, P. Puri, and P. K. Kythe, Some exact solutions of the Stokes problem for an elasticoviscous fluid, Acta Mech. 68 (1987), no. 3-4, 223-230.

[11] R. L. Fosdick and K. R. Rajagopal, Thermodynamics and stability of fluids of third grade, Proc. Roy. Soc. London Ser. A 369 (1980), no. 1738, 351-377.

[12] K. R. Frater, A boundary layer in an elastico-viscous fluid, Z. Angew. Math. Phys. 20 (1969), 712-721.

[13] V. K. Garg and K. R. Rajagopal, Flow of a non-Newtonian fluid past a wedge, Acta Mech. 88 (1991), no. 1-2, 113-123.

[14] T. Hayat, S. Asghar, and A. M. Siddiqui, Periodic unsteady flows of a non-Newtonian fluid, Acta Mech. 131 (1998), no. 3-4, 169-175.

[15] __ On the moment of a plane disk in a non-Newtonian fluid, Acta Mech. 136 (1999), no. 34, 125-131.

[16] Unsteady flow of an oscillating porous disk and a fluid at infinity, Meccanica 34 (1999), no. 4, 259-265.

[17] T. Hayat, M. R. Mohyuddin, S. Asghar, and A. M. Siddiqui, The flow of a viscoelastic fluid on an oscillating plate, Z. Angew. Math. Mech. 84 (2004), no. 1, 65-70.

[18] C.-H. Hsu and K.-L. Hsiao, Conjugate heat transfer of a plate fin in a second-grade fluid flow, Int. J. Heat Mass Transf. 41 (1998), no. 8-9, 1087-1102.

[19] R. R. Huilgol, Continuum Mechanics of Viscoelastic Liquids, Hindustan, Delhi, 1975.

[20] J. Málek, J. Nečas, M. Rokyta, and M. Růžička, Weak and Measure-Valued Solutions to Evolutionary PDEs, Applied Mathematics and Mathematical Computation, vol. 13, Chapman \& Hall, London, 1996.

[21] J. Málek, K. R. Rajagopal, and M. Růžička, Existence and regularity of solutions and the stability of the rest state for fluids with shear dependent viscosity, Math. Models Methods Appl. Sci. 5 (1995), no. 6, 789-812.

[22] D. Mansutti, G. Pontrelli, and K. R. Rajagopal, Steady flows of non-Newtonian fluids past a porous plate with suction or injection, Internat. J. Numer. Methods Fluids 17 (1993), 927941.

[23] K. R. Rajagopal, A note on unsteady unidirectional flows of a non-Newtonian fluid, Internat. J. Non-Linear Mech. 17 (1982), no. 5-6, 369-373. 
, Mechanics of non-Newtonian fluids, Recent Developments in Theoretical Fluid Mechanics (Paseky, 1992) (G. P. Gladi and J. Necas, eds.), Pitman Res. Notes Math. Ser., vol. 291, Longman Scientific \& Technical, Harlow, 1993, pp. 129-162.

[25] K. R. Rajagopal and A. S. Gupta, On a class of exact solutions to the equations of motion of a second grade fluid, Internat. J. Engrg. Sci. 19 (1981), no. 7, 1009-1014.

[26] An exact solution for the flow of a non-Newtonian fluid past an infinite porous plate, Meccanica 19 (1984), no. 2, 158-160.

[27] K. R. Rajagopal, A. S. Gupta, and T. Y. Na, A note on the Falkner-Skan flows of a non-Newtonian fluid, Internat. J. Non-Linear Mech. 18 (1983), no. 4, 313-320.

[28] K. R. Rajagopal and T. Y. Na, On Stokes problem for a non-Newtonian fluid, Acta Mech. 48 (1983), 233-239.

[29] G. K. Rajeswari and S. L. Rathna, Flow of a particular class of non-Newtonian visco-elastic and visco-inelastic fluids near a stagnation point, Z. Angew. Math. Phys. 13 (1962), 43-57.

[30] M. Reiner, Rheology, Handbuch der Physik (S. Flugge, ed.), vol. 6, Springer-Verlag, Berlin, 1958, pp. 434-550.

[31] R. S. Rivlin and J. L. Ericksen, Stress-deformation relations for isotropic materials, J. Rational Mech. Anal. 4 (1955), 323-425.

[32] W. R. Schowalter, Mechanics of Non-Newtonian Fluids, Pergamon, Oxford, 1978.

[33] A. M. Siddiqui, T. Hayat, and S. Asghar, Periodic flows of a non-Newtonian fluid between two parallel plates, Internat. J. Non-Linear Mech. 34 (1999), no. 5, 895-899.

[34] A. C. Srivastava, The flow of a non-Newtonian liquid near a stagnation point, Z. Angew. Math. Phys. 9 (1958), 80-84.

[35] C. Truesdell and W. Noll, The Nonlinear Field Theories of Mechanics, 2nd ed., Springer-Verlag, Berlin, 1992.

[36] S. Turbatu, K. Bühler, and J. Zierep, New solutions of the II. Stokes problem for an oscillating flat plate, Acta Mech. 129 (1998), no. 1-2, 25-30.

S. Asghar: Department of Mathematics, COMSATS Institute of Information Technology, 22010 Abbottabad, Pakistan

E-mail address: s_asgharpk@yahoo.com

M. R. Mohyuddin: Department of Mathematics, Quaid-i-Azam University, Islamabad 45320, Pakistan

E-mail address: m_raheel@yahoo.com

T. Hayat: Department of Mathematics, Quaid-i-Azam University, Islamabad 45320, Pakistan

E-mail address: t_pensy@hotmail.com

A. M. Siddiqui: Department of Mathematics, Pennsylvania State University, York Campus, York, PA 17403, USA

E-mail address: ams5@psu.edu 


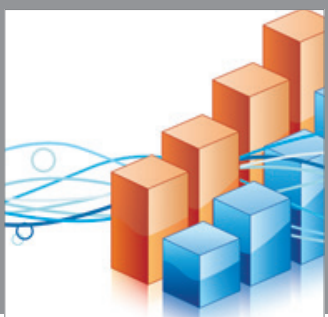

Advances in

Operations Research

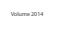

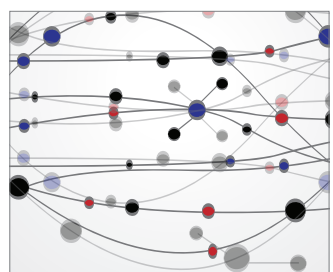

\section{The Scientific} World Journal
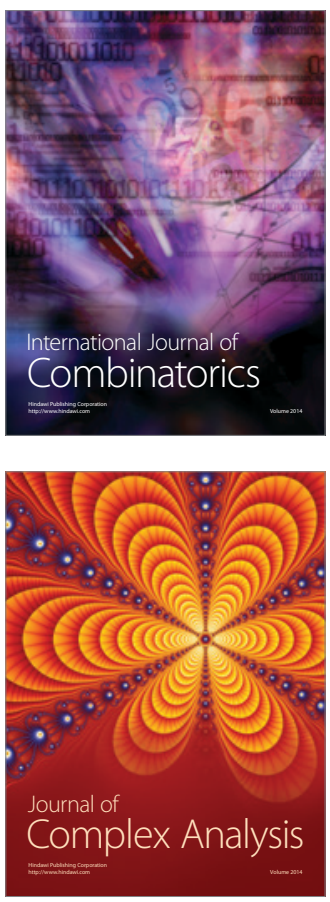

International Journal of

Mathematics and

Mathematical

Sciences
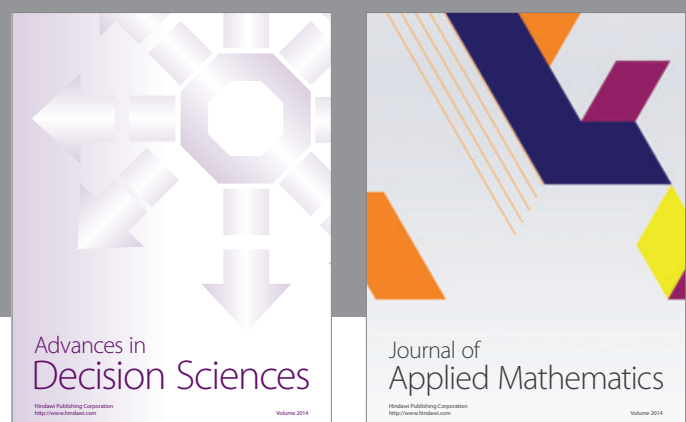

Journal of

Applied Mathematics
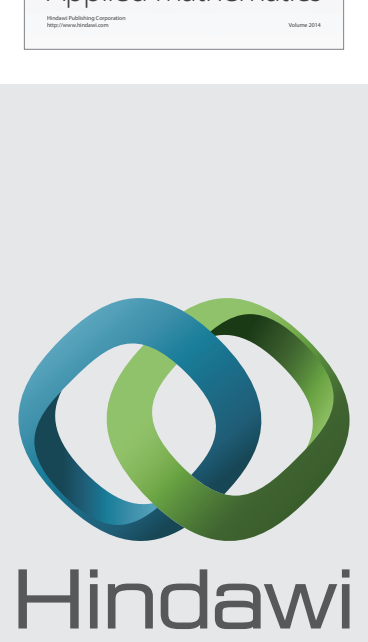

Submit your manuscripts at http://www.hindawi.com
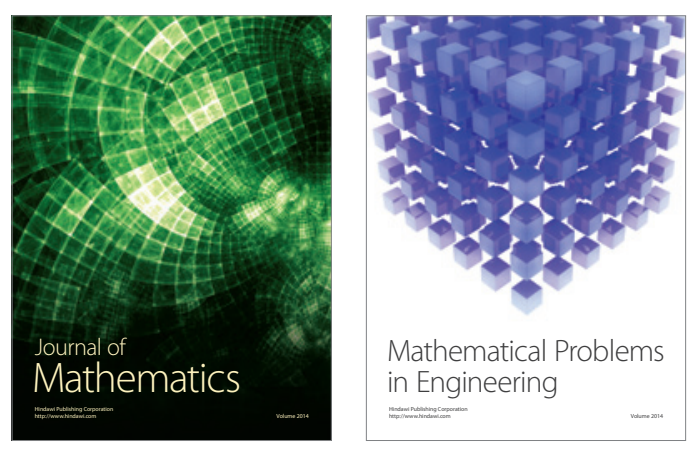

Mathematical Problems in Engineering
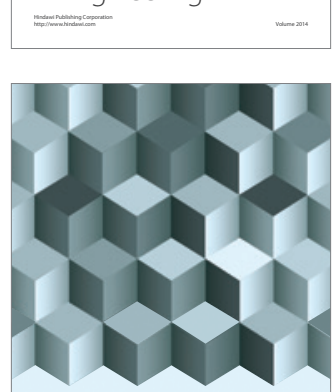

Journal of

Function Spaces
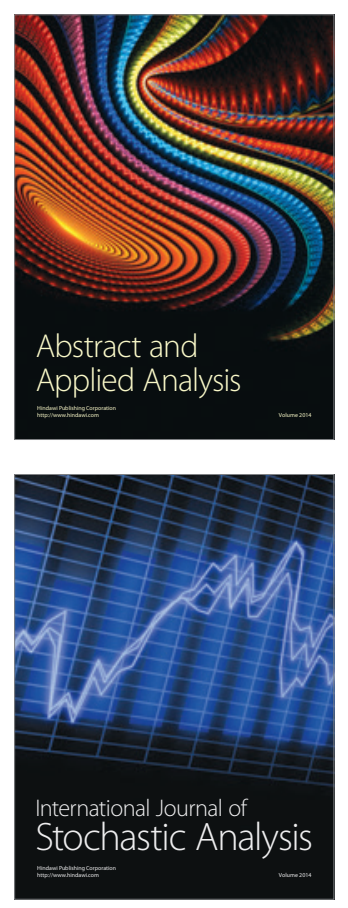

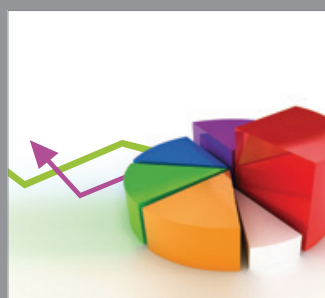

ournal of

Probability and Statistics

Promensencen
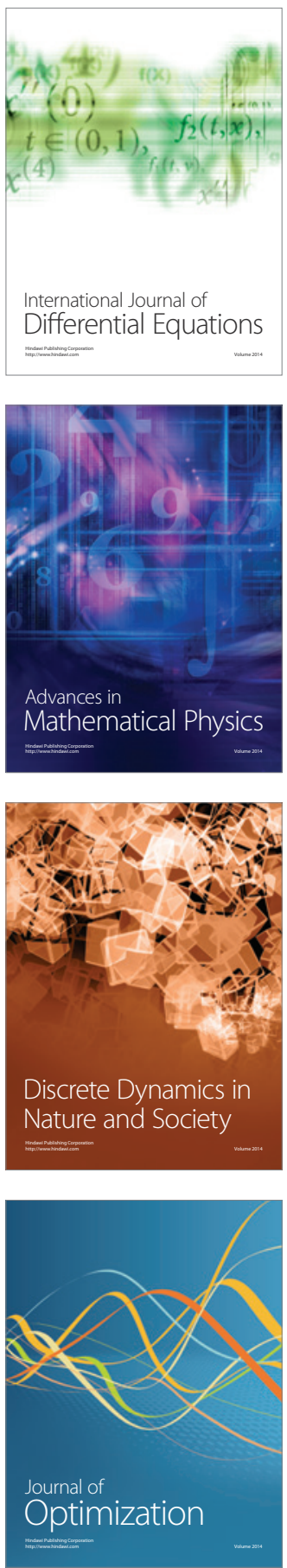